\title{
Action synchronization with biological motion
}

\author{
Lincoln J. Colling (lincoln.colling@maccs.mq.edu.au) \\ William F. Thompson (bill.thompson@mq.edu.au) \\ John Sutton (john.sutton@maccs.mq.edu.au)
}

Music, Sound and Performance Laboratory and Macquarie Centre for Cognitive Science

Macquarie University, Sydney NSW 2112 Australia

\begin{abstract}
The ability to predict the actions of other agents is vital for joint action tasks. Recent theory suggests that action prediction relies on an emulator system that permits observers to use information about their own motor dynamics to predict the actions of other agents. If this is the case, then predictions for self-generated actions should be more accurate than predictions for other-generated actions. We tested this hypothesis by employing a self/other synchronization paradigm where prediction accuracy for recording of self-generated movements was compared with prediction accuracy for other-generated movements. As expected, predictions were more accurate when the observer's movement dynamics matched the movement dynamics of the recording. This is consistent with that idea that the observer's movement dynamics influence the predictions they generate.
\end{abstract}

Keywords: Biological motion; action; perception

\section{Introduction}

In order to engage in joint action it is crucially important that agents are able to coordinate their actions with each other. Humans possess an astonishing ability for highly accurate temporal coordination of action. This ability for temporally coordinated, or synchronized, action is evident in pursuits such as music and dance, where synchronized action can be an important part of the aesthetic qualities of the performance. That humans can synchronize their actions with great accuracy is not in doubt; however, what is less clear is the mechanism that underlies this ability.

Synchronization tasks impose heavy time demands on action execution. To meet these time demands, actions need to be planned early so that execution can be temporally synchronized. Therefore, it is unlikely that actions are planned based on the incoming sensory input because sensory transduction would introduce disruptive time delays. To bypass these delays, and to achieve the temporal precision that is evident in many synchronization tasks, it is necessary for actors to anticipate the movements of their co-actors. This may be done by predicting how their co-actor might move. The results of these predictions would be available to the actor's action system before sensory input from the outside world and could, therefore, be used as the basis for early, or anticipatory, motor planning.

\section{Prediction}

Anticipatory motor planning, and prediction, has been extensively studied within the context of sensorimotor synchronization (SMS) tasks. These tasks often take the form of finger tapping in time with a regular beat (for a review, see Repp, 2005). In order to achieve synchronization in this type of task it is necessary to form a representation of the temporal structure of the stimulus so that the appropriate motor command can be issued before the next beat occurs. That is, it is necessary to take the current stream of sensory information and predict the future stream of sensory information. Recent neurophysiological evidence suggests a crucial role for the cerebellum in SMS tasks. This is unsurprising considering the role that the cerebellum plays in prediction, particularly during motor control (Miall et al., 1993).

Models of cerebellar function suggest that a core function of the cerebellum is to implement a forward, or predictive, model (Miall et al., 1993). Forward models use the current state of a system as input and from this they predict the future state of the system. This type of prediction may be particularly important during action control where the current state of an effector, along with the motor command, is used to predict the proprioceptive feedback that would be generated by a particular movement. This predicted proprioceptive feedback is thought to stand in for actual sensory feedback thereby bypassing sensory transmission delays (for a review, see Wolpert, Miall, \& Kawato, 1998). In SMS tasks, forward models may also play a similar role in anticipating future sensory events by taking the incoming stream of sensory events (beats) and projecting it forward in time (Tesche \& Karhu, 2000).

Predicting Human Action SMS tasks, such as tapping to the beat, require people to predict temporal properties of stimuli that are often relatively artificial. There has only been limited to work to extend the SMS paradigm into more naturalistic settings. For example, Repp and Keller (2008) employed a tapping paradigm that simulated tapping in time with another human subject. This use of more human-like stimuli is important because there is increasing evidence that stimuli generated by human movement are perceptually special. For example, it has been shown that the motor laws 
that govern the production of movements also influence how these movements are subsequently perceived. For example, the two-thirds power law relates the speed at which a curved trajectory is drawn to the curvature of the trajectory - that is, it is a law governing action production (Viviani \& Terzuolo, 1982). This law, however, also influences perceptual tasks. For example, Viviani and Stucchi, (1992) found that when subjects viewed pen traces producing an ellipse, and were asked to adjust the velocity profile of the traces so that they appeared to move around the ellipse at a constant speed, subjects made systematic errors and selected velocity profiles that were consistent with the two-thirds power law. Thus, for these stimuli, there is evidence that motor processes "leak" into, or affect, perceptual processes.

The two-thirds power law also influences the ability to generate predictions about the stimuli. Kandel, Orliaguet, and Viviani (2000) asked subjects to view excerpts of pen trajectories that were instances of either the letter combination "l1l" or "lln" written in a cursive style. When shown only the middle letter, and then asked to predict whether the following letter would be an "l" or an " $n$ ", observers were able to perform this task with reasonable accuracy (approximately $69 \%$ correct). When the trajectories were altered, however, so that the velocity profile of the trajectory no longer approximated real handwriting (that is, when it no longer obeyed the two-thirds power law) the accuracy with which the last letter could be predicted dropped markedly.

It is unsurprising that motor processes can influence perceptual processes for human-movement produced stimuli considering neurophysiological evidence suggests a tight link between perceptual and motor processes. In particular, mirror neurons $(\mathrm{MN})$ and canonical neurons in premotor cortex have been shown to have both perceptual and motor properties (for a review, see Gallese et al., 1996). The MN system transforms perceptual information into motor information by being active both when a particular action is performed and when that action is observed (or when a sound associated with the action is heard; Kohler et al., 2002). This suggests that there may be a common neural code for the production and perception of actions. A number of proposals have been put forward for the role that MNs might play in human cognition. Most prominent among these is the notion that MNs might be the neural substrate of third person mind reading (e.g., Vittorio Gallese \& Goldman, 1998). However, some have criticized this view and an alternative view, that MNs might underlie the more basic function of predicting the actions of other agents, has been suggested (Jacob, 2008; Wilson \& Knoblich, 2005).

\section{The Emulator Model of Human Action Prediction}

Wilson and Knoblich (2005) have suggested that MNs form part of an emulator, or forward model (Grush, 2004), that is used to predict the movements of other agents. They suggest that the emulator generates a real-time simulation of the movements of other agents, and these simulations, which run concurrently with sensory input, are then used to predict the unfolding trajectory of an observed motor act. These predictions are able to stand in for actual sensory input bypassing delays that would be introduced by waiting for actual sensory information to arrive. Therefore, predicted input can serve as the basis for early, or anticipatory, motor planning. It is unlikely that the emulator is instantiated in a purely perceptual system because of the computational complexity of predicting human movement. This computational challenge may be greatly reduced if the emulator piggybacks on the motor system, by way of MNs, thus allowing the emulator to exploit existing knowledge of human movement dynamics that is embodied in the motor system.

If it is the case that knowledge embodied in the observer's motor system is used as the basis for predicting the actions of other agents, then idiosyncrasies of the observer's motor system should "leak out" into the predictions that they generate. It follows that the emulator hypothesis would predict superior prediction accuracy when observers generate predictions about the movements of agents that move similarly to themselves. That is, if observers use knowledge of their own motor dynamics to generate predictions about how other agents will move then a better match between the movement dynamics of the observer and the observed agent will result in enhanced prediction accuracy. This should be most evident when observers generate predictions about their own (recorded) movements, because in this case there is a perfect match between the dynamics of the predicting system and the predicted system. This hypothesis has found support in several studies using a self/other prediction paradigm.

The self/other prediction paradigm employed by Knoblich and Flach (2001) required observers to generate a prediction about the landing position of a dart that was thrown at a dartboard. Observers were shown a series of video recordings depicting both the observer and another person throwing darts towards a dartboard. The recordings were stopped at the point where the dart was released from the hand, and the observer then had to generate a prediction about the landing position. Observers were significantly better at predicting the dart landing position from video recordings of themselves compared to recordings of other people. A similar experiment by Knoblich et al. (2002) asked subjects to generate a prediction about whether a pen stroke was produced in isolation or as part of a character. Once again, predictions were more accurate when observers viewed their own handwriting.

The studies outlined above are consistent with a role for motor emulation in action prediction; however, these studies employed an offline (or observe then predict) prediction paradigm where subjects generated their prediction offline after viewing the stimulus. It is still not clear how rapidly the information from the emulator is available to use for online action control. To assess this, Flach, Knoblich, and Prinz (2003) employed a self/other synchronization task. This task required subjects to observe a moving dot as it traced a zigzag or wave trajectory (cf. Figure 1), and to press a response button when the moving dot reached the apex of each peak. These trajectories had been generated 

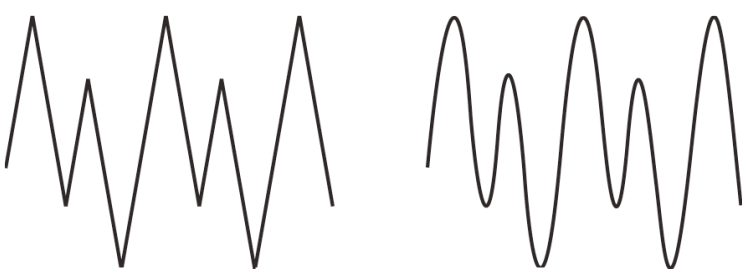

Figure 1: The zigzag (left) and wave (right) patterns used as stimuli during the recording session.

earlier by asking subjects to draw them on a digitizer tablet. During the test session, subjects were presented with trajectories that they had produced themselves as well as trajectories that had been produced by another person. As predicted by the emulator hypothesis, superior prediction accuracy (as measured by synchronization error) was observed for selfproduced trajectories over other-produced trajectories. However, this self-prediction advantage, or authorship effect, only emerged in the final two (of four) experimental blocks ${ }^{1}$.

The delay in the onset of the authorship effect, observed by Flach et al. (2003), is surprising because Knoblich and Flach (2001) were able to observe an immediate authorship effect in their dart throwing experiment. The most salient difference between Knoblich and Flach (2001) and Flach et al. (2003) is that Knoblich and Flach presented observers with actual bodily information (information about the limbs and joints) whereas Flach et al. only presented observers with the consequences of human movement, namely pen traces. It may take time for observers to map these motor consequences onto their own action system before they can exploit the knowledge embodied in their action system for the purposes of synchronization. If this hypothesis is correct, then it may be possible to achieve an immediate authorship effect by presenting bodily movement to observers.

\section{Aims and Hypotheses}

The primary aim of the present study is to replicate the findings of Flach et al. (2003) which demonstrate that observers use knowledge embodied in their own action system in order to predict how other agents will move. Furthermore, we aim to extend these findings by using stimuli that include actual bodily movements rather than stimuli that merely show the consequences of human movements. To this end, we employ moving animations of 3-D characters as stimuli. We anticipate that prediction accuracy (as measured by syn-

\footnotetext{
${ }^{1}$ Within the auditory domain, Keller, Knoblich, and Repp (2007) asked pianists to play duets with either recordings themselves or another person, and superior timing accuracy was observed when pianists accompanied recordings of their own playing. However, the movement durations were relatively long (70s) and the authors provide only timing errors averaged over the entire piece. Therefore, it is difficult to assess whether the authorship effect emerged over time.
}

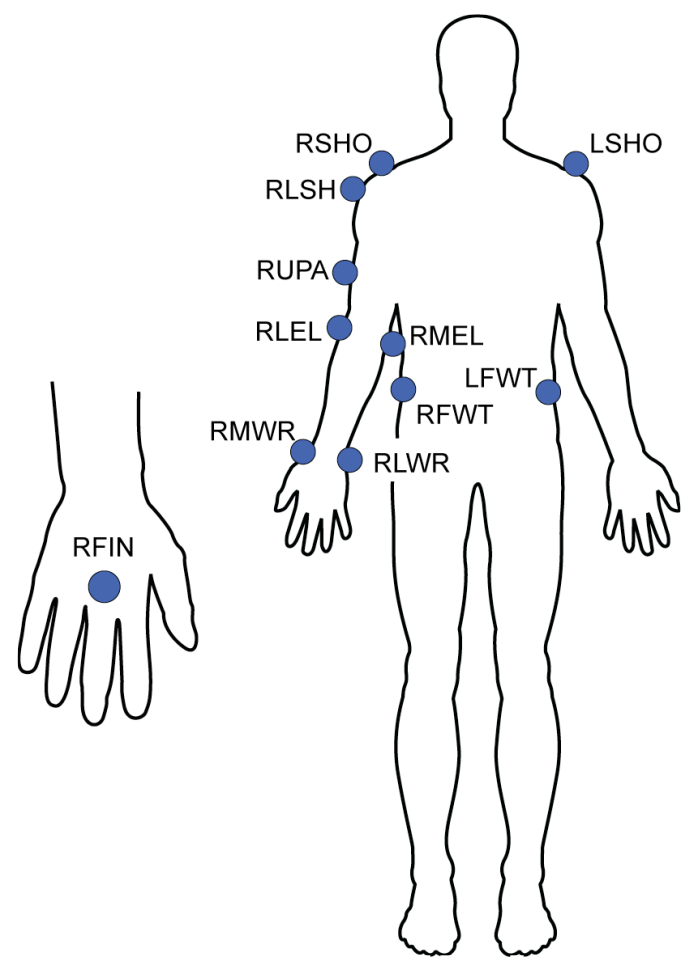

Figure 2: Marker positions for the 11 reflective markers used during the recording session.

chronization accuracy) will be enhanced when observers synchronize with 3-D characters constructed from selfgenerated movements compared with characters constructed from other-generated movements. Furthermore, we predict that the inclusion of actual bodily information (in the form of limbs and joints) will result in an immediate authorship effect.

\section{Methods and Materials}

\section{Subjects}

Twenty-two subjects (18 females), with an average age of 24 years (range: 18 to 49 years), took part in the experiment. Twenty-one subjects were right handed as assessed by the Edinburgh Handedness Inventory (Oldfield, 1971). All subjects had normal or corrected-to-normal vision and were free of movement difficulties. All procedures were approved by the Macquarie University Human Ethics Committee.

\section{Motion Capture}

Motion data was recorded using an 8-camera 3-D passive optical motion capture system (Vicon MX+ with 4 Vicon MX-F20 and 4 Vicon MX13+ cameras) at a sampling rate of $200 \mathrm{~Hz}$. A total of 11 spherical markers measuring $9 \mathrm{~mm}$ in diameter were placed on the shoulders, right arm, right hand, and waist of the subject (see Figure 2). The shoulder markers were placed on the top of the left shoulder (LSHO) and right shoulder (RSHO) and on the lateral side of the right shoulder (RLSH). Right arm markers were placed on 


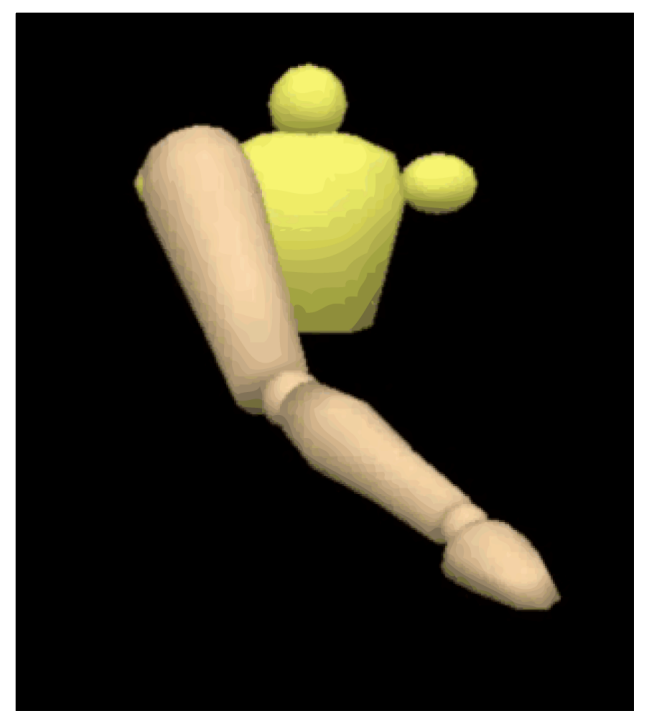

Figure 3: Sample of the 3-D animated character used during the test session.

the lateral side of the right upper arm (RUPA) and on the inside (RMEL) and outside (RLEL) elbow. Right hand markers were placed on the medial wrist (RMWR) and lateral wrist (RLWR) and at the base of the right middle finger (RFIN). Waist markers were placed between the iliac crest and the rib cage on the left (LFWT) and right (RFWT) side. Subjects were instructed to keep their left arm rested by their side to ensure that it did not obscure the left waist marker. Subjects were also instructed to clench their fist to ensure that the fingers did not move independently of the rest of the hand.

\section{Stimuli}

Recording Session Subjects reproduced two movement patterns (Figure 1, adapted from Flach et al., 2003) during the recording session. These movement patterns were chosen so that the results of the present study would be more directly comparable to those of previous studies (i.e., Flach et al., 2003). Both patterns consisted of five peaks and five troughs with the peaks alternating in height. The two patterns differed, however, in the nature of the direction change at the apex of the peaks. The zigzag pattern had a sharp direction change while the wave pattern had a smooth, flowing, direction change. Subjects viewed the patterns on large sheets of cardboard measuring $0.594 \mathrm{~m} \times 0.841 \mathrm{~m}$. For the zigzag pattern, the first up-stroke was $0.3 \mathrm{~m}$ long, with the remaining short up-strokes measuring $0.25 \mathrm{~m}$ and the long up-strokes measuring $0.5 \mathrm{~m}$. All the down-strokes were $0.375 \mathrm{~m}$ long. The angles enclosed by the strokes measured 0.35 radians. The wave pattern was based on the zigzag pattern and the stroke lengths were closely matched. The eccentricity of the curve at the apex was approximately 0.95 for all peaks.
Test Session Raw motion capture data was first resampled to $25 \mathrm{~Hz}$. Animated characters were constructed with $\mathrm{C}$ Motion Visual 3D (C-Motion INC, Rockville MD) using the resampled motion data. The characters consisted of an upper torso and right arm and hand. The torso was constructed using the shoulder markers (RSHO and LSHO) and waist markers (RFWT and LFWT). The upper arm was constructed using the shoulder markers and elbow markers (RMEL and RLEL), and the right lower arm was constructed using the elbow and wrist markers (RMWR and RLWR). The hand was constructed using the wrist markers as the proximal endpoint and the right finger marker (RFIN) as the midpoint. The size of the torso was not accurately modeled for each subject; however, the limb lengths were modeled using measurements acquired from the captured marker positions. Thus, limb lengths appeared relatively longer for animations constructed from taller subjects and shorter for animations constructed from shorter subjects. All animated characters were presented from a frontal view so that the action appeared as if viewed from the front (See Figure 3).

\section{Procedure}

The experiment was split over two sessions separated by approximately $2-6$ weeks $(\mathrm{M}=18$ days, $\mathrm{SD}=7)$. The delay was introduced to control for possible effects of episodic memory recall. During the recording session, subjects sat on a chair in the middle of the capture volume. At the start of each trial, they were shown the appropriate pattern and were told to produce either a wave or a zigzag. To generate the movements, subjects were asked to pretend that they were drawing the patterns on a large blackboard. This involved making a series of up-and-down arm movements. All subjects produced the movements with their right hand. Subjects practiced each pattern once with their eyes open and then reproduced each pattern 15 times. Subjects performed these movements with their eyes closed to control for visual familiarity with the movement. The movements were produced in 3 blocks containing 5 repetitions of each pattern in a randomized order. Subjects reproduced the patterns at their own pace (see Movement Description for details of intersubject variability).

In the test session, subjects observed a series of animated 3-D characters (see Figure 3). Animations were used in order to control for the possible effects of overt recognition cues. The movement of the characters was controlled by the motion capture data recorded during the recording session. To ensure that stimulus factors did not influence synchronization accuracy, subjects were grouped into randomly assigned pairs, and each subject observed a series of animated characters controlled by their own movement data as well as a series of animated characters controlled by the movement data of the other subject in the pair. Both subjects in the pair viewed the same set of animations in identical order. The task in the test session was to press the response button when the hand of the character reached the peak of each upward movement. Subjects were instructed to synchronize 


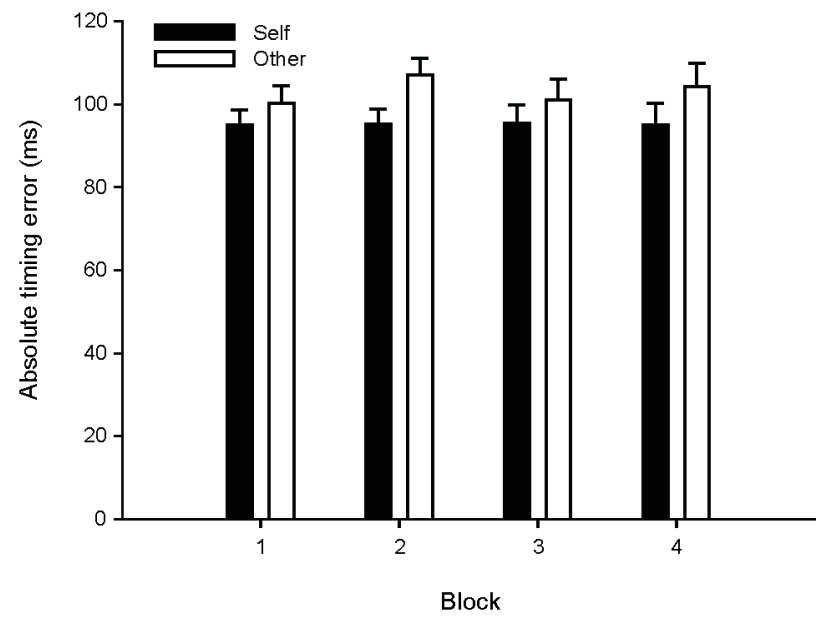

Figure 4: Mean timing error for self-generated and other-generated actions (error bars indicate SE).

the button press with the display as accurately as possible and were told that this may require them to anticipate when the peak will occur. Each subject performed 4 blocks containing 40 unique stimuli (20 trials of self-generated movements and 20 trials of other-generated movements). The stimuli were presented in a pseudo-random order so that self-generated movements and other-generated movements were evenly distributed through the block.

\section{Movement Description}

The length of each movement (distance traveled by the RFIN marker) was on average $4.051 \mathrm{~m}(\mathrm{SD}=0.570)$ with subject averages ranging between $3.208 \mathrm{~m}$ and $5.487 \mathrm{~m}$. The average length of the long up-strokes was $0.441 \mathrm{~m}$ ( $\mathrm{SD}=0.066$; range: $0.611 \mathrm{~m}$ to $0.355 \mathrm{~m}$ ), and the average length of the short up-strokes was $0.315 \mathrm{~m}(\mathrm{SD}=0.061$; range: $0.435 \mathrm{~m}$ to $0.230 \mathrm{~m}$ ). The average length of the down-strokes was $0.414 \mathrm{~m}(\mathrm{SD}=0.060$; range: $0.557 \mathrm{~m}$ to $0.332 \mathrm{~m}$ ). The average duration of each movement was $6.86 \mathrm{~s}(\mathrm{SD}=1.74)$ with subject averages ranging between $3.37 \mathrm{~s}$ and $10.05 \mathrm{~s}$. All movements were completed in less than $12 \mathrm{~s}$. The average up-stroke duration was $0.66 \mathrm{~s}$ $(\mathrm{SD}=0.16)$ for the long up-stroke and $0.59 \mathrm{~s}(\mathrm{SD}=0.16)$ for the short up-stroke. The average duration for the downstrokes was $0.71 \mathrm{~s}(\mathrm{SD}=0.19)$.

\section{Results}

The peak of each upward movement was determined using the raw motion capture data. The movement leading up to the first peak was often contaminated with artifact and, therefore, the first peak was dropped from analysis. Timing error was calculated as the absolute difference between the timing of the peak in the motion capture data and the timing of the button press. Button presses with a timing error that was greater than $250 \mathrm{~ms}$ were deemed too slow and were excluded from analysis. This was done to ensure that sub- jects were anticipating the peak rather than merely responding to the peak once it had appeared on screen. This resulted in approximately $9.8 \%$ of button presses being dropped from analysis (including all button presses did not change the pattern of results reported below). Data analysis was performed on absolute timing error using a $2 \times 4$ repeated measures ANOVA with the factors Authorship (self, other) and Block (1, 2, 3, and 4).

We found a significant main effect of Authorship $[F(1,21)=6.266, p=.021]$ indicating that timing error was significantly lower when synchronizing with recordings of self-generated actions $(\mathrm{M}=95 \mathrm{~ms}, \mathrm{SD}=20)$ compared to other-generated actions $(\mathrm{M}=103 \mathrm{~ms}, \mathrm{SD}=22)$. The authorship effect, calculated as the difference in timing error between other-generated and self-generated actions, was positive (i.e., higher timing error for other-generated actions) for 10 of the 11 subject pairs. The main effect for Block $[\mathrm{F}(3,63)=0.354, \mathrm{p}=.786]$ and the Block $\times$ Authorship $[\mathrm{F}(3,63)=1.708, \mathrm{p}=.174]$ interaction did not reach significance, indicating that both the timing error and the size of the authorship effect did not vary systematically across the four experimental blocks. These data are shown in Figure 4.

Previous studies (for a review, see Fraisse, 1982) have suggested that people have a preferred tempo for producing rhythmic movements such as the arm movements and the button press movements in the present study. Therefore, it is possible that the authorship effect is not related to motor emulation but is rather dependent on there being a difference in the preferred tempo between the two subjects in the pair. To test this possibility, we examined the relationship between the size of the authorship effect and the tempo similarity of the two subjects in the pair. Tempo similarity was measured as the absolute difference in average tempo (measured in peaks per minute) for the arm movement recordings from each subject in the pair. No reliable relationship was found between the size of the authorship effect and tempo similarity $(r=-.040, p=.909)$. These data are shown in Figure 5 (top).

The results of Flach et al. (2003) have suggested that the authorship effect is dependent on peak irregularity. An authorship effect was not found for movements where the peak height did not alternate between large and small. To test for an effect of peak irregularity in the present data, we examined the relationship between the size of the authorship effect and peak irregularity (measured as the absolute difference between the average length of the large up-strokes and the average length of the small up-strokes). An increase in peak irregularity was associated with an increased authorship effect (see Figure 5 bottom). However, this relationship only approached significance $(\mathrm{r}=.548, \mathrm{p}=.081)$.

\section{Discussion}

The primary aim of the present study was to investigate the mechanisms underlying interpersonal action prediction. In particular, we tested the hypothesis that observers use knowledge embodied in their own action system to generate predictions about the movements of other agents. Our re- 

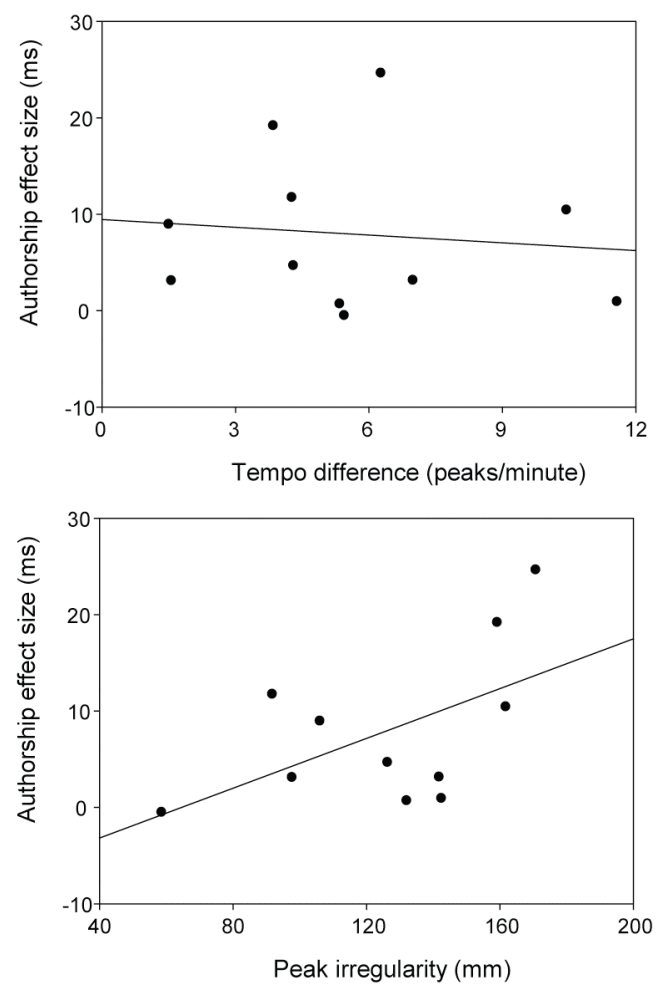

Figure 5: The relationship between the size of the authorship effect and tempo difference (top) and the size of the authorship effect and peak irregularity (bottom).

sults are consistent with the hypothesis that prediction accuracy is dependent on the match between the observer's and observed agent's action systems.

\section{Authorship Effect}

In order to measure the degree to which the observer's action system influenced their ability to predict the actions of other agents, we measured prediction accuracy (measured as synchronization accuracy) for displays of self-generated and other-generated actions. The logic being that if the observer used knowledge embodied in their own action system to predict the actions of other agents then prediction accuracy should be enhanced when there is a perfect match between the system used for predicting and the system being predicted - that is, when observing recordings of self-generated action. As predicted, synchronization accuracy was enhanced when subjects synchronized with recordings of selfgenerated action compared with recordings of othergenerated actions. This is consistent with the idea that observers use their own motor dynamics as the basis for action prediction. Our findings are consistent with previous experiments using similar stimuli (Flach et al., 2003).

An alternative explanation for the results is that subjects found it easier to synchronize with recordings of selfgenerated actions because these actions were produced at a comfortable tempo for producing button presses. Rather than attempting to predict the peak of each upward move- ment, subjects may have merely produced button presses at a tempo comfortable for them, and synchronization accuracy was enhanced due to the coincidence between their preferred tempo for producing arm movements and their preferred tempo for producing button presses. Several studies (for a review, see Fraisse, 1982) have suggested that people do indeed have a preferred tempo for spontaneous rhythmic movement (although there is little evidence that preferred tempo is consistent across different effectors). Because we did not control for production tempo, it is therefore possible that subjects produced arm movements at their preferred tempo. Furthermore, the use of regular rhythmic stimuli may have enhanced the tendency to produce movements at a preferred tempo. Synchronization accuracy for self-generated actions may have been enhanced merely by reproducing their own preferred tempo during the test session. We attempted to rule out this possibility by examining the relationship between the size of the self-synchronization advantage (or authorship effect) for the two subjects in the pair and the similarity between arm movement tempo for the two subjects in the pair. If these results could be explained by preferred tempo then we would expect to find that the authorship effect increased as the difference in arm movement tempo increased. This, however, was not the case, and there was no systematic relationship between tempo difference and the size of the authorship effect.

We also tested for a relationship between the size of the authorship effect and the regularity of the peak heights. Flach et al. (2003) found that the authorship effect was only present when subjects synchronized with irregular movements but not with regular movements. The authors suggested that when the movements were regular the subjects could use the height of the preceding peak as a prediction cue for the height of the following peak. Therefore, the height of the following peak could be predicted without the need to rely on knowledge embodied in the observer's action system.

In the case of irregular movements, however, the height of the preceding peak cannot be used as a cue for the height of the following peak. Therefore, subjects may need to make use of knowledge embodied in their own action system (that is, motor emulation) in order to predict the height of the following peak. The results of the present study are suggestive of a relationship between movement irregularity and the size of the authorship effect with the authorship effect increasing as movements become more irregular. The alternative hypothesis - that enhanced synchronization accuracy is due to the coincidence of idiosyncratic tempo-would not predict any effect of peak irregularity. Future experiments will need to explicitly rule out this alternative explanation by either explicitly controlling for production tempo or by using non-rhythmic stimuli.

A further alternative explanation for the results presented here is that superior timing accuracy was observed for selfgenerated movements because subjects had more visual experience with their own movements. On this account, the authorship effect would be due to purely perceptual proc- 
esses. However, the up-and-down arm movements employed in our task are unlikely to be movements that are commonly performed and, therefore, it is unlikely that subjects have acquired visual experience with them. Furthermore, during the recording session, subjects performed the movements with their eyes closed ruling out the possibility of acquiring visual experience during the experiment. It is more plausible that subjects acquired motor experience during the recording session, and the use of this motor knowledge for action prediction is consistent with the emulator hypothesis.

\section{Block Effect}

Flach et al. (2003, experiment 2), in a study measuring synchronization accuracy with up-and-down pen traces, found no difference between synchronization accuracy for selfgenerated and other-generated actions during the first two experimental blocks. Only during the third and fourth experimental block did the authorship effect emerge. In the present study, however, there was no systematic change in the size of the authorship effect across the four experimental blocks. The most salient difference between Flach et al. and the present study is that Flach et al. did not present subjects with actual human movement. The pen traces used as stimuli in this study were representations of the results or consequences of human movement. The present study, however, presented subjects with actual limb movements by using 3$\mathrm{D}$ animated characters composed of a torso and right arm. Stimuli of this type are isomorphic with the observer and, therefore, may be more easily mapped onto the observer's body. Before the emulator system can be used for action prediction, the emulator needs information about which effectors to model, and providing this information in the stimulus may facilitate building an appropriate model. The somatatopic organization of the MN system (Buccino et al., 2001) suggests that stimuli of this type may lead to increased MN activation in the observer. This increase in somatatopic activation may be what is providing an increase in information about the initial parameter settings (e.g., which limbs to model) to the observer's emulator system and this may enhance motor emulation. The authorship effect arises because the emulator uses the observer's own motor dynamics to model the observed agent and, therefore, enhancing motor emulation would also lead to an enhancement of the authorship effect. In the case of pen traces an extra step is required whereby the consequences of movement (i.e., the pen traces) would need to be mapped onto those effectors that are responsible for producing that movement. Before this step occurs the emulator will not have access to information about which effectors movements to model, and this would reduce the influence that the observer's motor dynamics have on their predictions (the authorship effect). This mapping step may take extra time because the relevant effectors are not actually present in the stimulus, and these mappings may need to be learned through the course of the experiment. The presence of this extra step in the experiments by Flach et al. and the absence of this extra step in the present study may account for our failure to replicate the block effect observed by Flach et al.

\section{Conclusions}

Taken together, the results of the present study suggest that observers generate predictions of how other agents will move based on information about how they themselves move. This is consistent with the emulator hypothesis of action prediction whereby observers' action systems form part of a forward model for interpersonal action prediction.

Future studies will need to rule out the possibility that these results can be explained by the coincidence of idiosyncratic tempo, and will need to address the exact role that bodily information might play in action prediction. Furthermore, the exact neural substrate of the emulator has not been investigated. If, as Wilson and Knoblich (2005) suggest, the emulator is instantiated in the human MN system then it may be possible to employ the stimuli used in the present study to investigate differences in MN activity that correlate with prediction accuracy. These stimuli are well suited to this task due to the somatatopic nature of the MN system and because these stimuli avoid the block effect observed in previous studies.

\section{Acknowledgments}

We thank Rachel Bennetts and Bojan Neskovic for assistance with data collection. LJC is supported by an MQRES scholarship.

\section{References}

Buccino, G., Binkofski, F., Fink, G. R., Fadiga, L., Fogassi, L., Gallese, V., et al. (2001). Action observation activates premotor and parietal areas in a somatotopic manner: an fMRI study. European Journal of Neuroscience, 13, 400404.

Flach, R., Knoblich, G., \& Prinz, W. (2003). Off-line authorship effects in action perception. Brain and Cognition, 53, 503-513.

Fraisse, P. (1982). Rhythm and tempo. In D. Deutsch (Ed.), The Psychology of Music (pp. 149-180). New York: Academic Press.

Gallese, V., Fadiga, L., Fogassi, L., \& Rizzolatti, G. (1996). Action recognition in the premotor cortex. Brain, 119, 593-609.

Gallese, V., \& Goldman, A. (1998). Mirror neurons and the simulation theory of mind-reading. Trends in Cognitive Sciences, 2, 493-501.

Grush, R. (2004). The emulation theory of representation: Motor control, imagery, and perception. Behavioral and Brain Sciences, 27, 377-396.

Jacob, P. (2008). What do mirror neurons contribute to human social cognition? Mind \& Language, 23, 190-223.

Kandel, S., Orliaguet, J. P., \& Viviani, P. (2000). Perceptual anticipation in handwriting: The role of implicit motor competence. Perception \& Psychophysics, 62, 706-716.

Keller, P. E., Knoblich, G., \& Repp, B. H. (2007). Pianists duet better when they play with themselves: On the possi- 
ble role of action simulation in synchronization. Consciousness and Cognition, 16, 102-111.

Knoblich, G., \& Flach, R. (2001). Predicting the effects of actions: Interactions of perception and action. Psychological Science, 12, 467-472.

Knoblich, G., Seigerschmidt, E., Flach, R., \& Prinz, W. (2002). Authorship effects in the prediction of handwriting strokes: Evidence for action simulation during action perception. The Quarterly Journal of Experimental Psychology: Section A, 55, 1027-1046.

Kohler, E., Keysers, C., Umilta, M. A., Fogassi, L., Gallese, V., \& Rizzolatti, G. (2002). Hearing sounds, understanding actions: Action representation in mirror neurons. Science, 297, 846-848.

Miall, R. C., Weir, D., Wolpert, D., \& Stein, J. (1993). Is the cerebellum a smith predictor? Journal of Motor Behavior, 25, 203-216.

Oldfield, R. C. (1971). The assessment and analysis of handedness: the Edinburgh inventory. Neuropsychologia, 9, 97-113.

Repp, B. H. (2005). Sensorimotor synchronization: a review of the tapping literature. Psychonomic Bulletin \& Review, 12, 969-992.

Repp, B. H., \& Keller, P. E. (2008). Sensorimotor synchronization with adaptively timed sequences. Human Movement Science, 27, 423-456.

Tesche, C. D., \& Karhu, J. J. (2000). Anticipatory cerebellar responses during somatosensory omission in man. Human Brain Mapping, 9, 119-142.

Viviani, P., \& Stucchi, N. (1992). Biological movements look uniform: evidence of motor-perceptual interactions. Journal of Experimental Psychology. Human Perception and Performance, 18, 603-623.

Viviani, P., \& Terzuolo, C. (1982). Trajectory determines movement dynamics. Neuroscience, 7, 431-437.

Wilson, M., \& Knoblich, G. (2005). The case for motor involvement in perceiving conspecifics. Psychological Bulletin, 131, 460-473.

Wolpert, D. M., Miall, R. C., \& Kawato, M. (1998). Internal models in the cerebellum. Trends in Cognitive Sciences, 2, 338-347.

\section{Citation details for this article:}

Colling, L., Thompson, W., Sutton, J. (2010). Action synchronization with biological motion. In W. Christensen, E. Schier, and J. Sutton (Eds.), ASCS09: Proceedings of the 9th Conference of the Australasian Society for Cognitive Science (pp. 49-56). Sydney: Macquarie Centre for Cognitive Science.

DOI: $10.5096 /$ ASCS20098

URL:

http://www.maccs.mq.edu.au/news/conferences/2009/ASCS 2009/html/colling thompson.html 